\title{
Domestic Pig Unlikely Reservoir for MERS-CoV
}

\section{Emmie de Wit, Friederike Feldmann, Eva Horne, Cynthia Martellaro, Elaine Haddock, Trenton Bushmaker, Kyle Rosenke, Atsushi Okumura, Rebecca Rosenke, Greg Saturday, Dana Scott, Heinz Feldmann}

We tested the suitability of the domestic pig as a model for Middle East respiratory syndrome coronavirus (MERS-CoV) infection. Inoculation did not cause disease, but a low level of virus replication, shedding, and seroconversion were observed. Pigs do not recapitulate human MERS-CoV and are unlikely to constitute a reservoir in nature.

\begin{abstract}
A s of March 10,2017, a total of 1,917 cases of Middle East respiratory syndrome coronavirus (MERS-CoV) infection and 684 fatalities have occurred (1). Despite the relatively large number of cases, little is known about the disease pathology of MERS in humans (2). Our current understanding of the pathogenesis of MERS-CoV is therefore mostly based on data derived from studies in animal models. Although the first animal model used to study MERS$\mathrm{CoV}$ pathogenesis and test potential countermeasures became available shortly after the discovery of MERS-CoV (3), all the animal models that have been developed so far have drawbacks (4). Because of the host restriction conferred by the binding of the MERS-CoV spike protein to its receptor, dipeptidyl peptidase 4 (DPP4), small animal models that are routinely used to conduct infectious disease research are not naturally susceptible to MERS-CoV infection. Although human DPP4-transgenic mouse models have been developed, these do not completely recapitulate the disease pathology observed in humans. Nonhuman primate models recapitulate mild and moderate human disease pathology; however, practical and ethical constraints limit work with these models.

The domestic pig (Sus domesticus) is used in infectious disease research because of similarities between human and pig anatomy, genetics, and physiology (5). MERS$\mathrm{CoV}$ was previously shown to replicate in porcine kidney cells, albeit less efficiently than in human kidney cells (6). In an effort to develop a MERS-CoV animal model that recapitulates human disease better than small animal models

Author affiliations: National Institutes of Health, Hamilton, Montana, USA (E. de Wit, F. Feldmann, E. Horne, C. Martellaro,

E. Haddock, T. Bushmaker, K. Rosenke, R. Rosenke, G. Saturday, D. Scott, H. Feldmann); Columbia University, New York, New York, USA (A. Okumura)
\end{abstract}

DOI: http://dx.doi.org/10.3201/eid2306.170096 without the constraints associated with nonhuman primate studies, we explored the possibility of using the domestic pig as an animal model of MERS-CoV infection.

\section{The Study}

Comparison of the DPP4 nucleotide sequences of humans, dromedary camels, and domestic pigs showed that the porcine DPP4 is identical to the dromedary camel DPP4 at the 14 aa positions that have been shown to determine species tropism (Table) $(8,9)$. We investigated whether DPP4 is expressed in the pig respiratory tract by performing immunohistochemical staining on the nasal mucosa and lung tissue obtained from healthy pigs using an antibody against DPP4 (mouse monoclonal anti-DPP4 [CD26], clone OTI11D7, 1:2,500; Origene Technologies, Inc., Rockville, MD, USA). DPP4 expression was not observed in the nasal mucosa of healthy domestic pigs (Figure 1, panel A); in the lungs, abundant DPP4 expression was observed in type I and type II pneumocytes and submucosal glands (Figure 1, panel B), suggesting MERS-CoV infection would be supported.

We inoculated 2 groups of four 4-5-week-old farm pigs (Yorkshire cross; S\&S Farms, Ramona, CA, USA) intranasally $(1 \mathrm{~mL} /$ nostril $)$ and intratracheally $(5 \mathrm{~mL})$ with a total dose of $10^{6}$ tissue culture infectious dose $50\left(\mathrm{TCID}_{50}\right)$ of the hCoV-EMC/2012 isolate of MERS-CoV. A group of 3 control pigs was mock inoculated with Dulbecco's modified Eagle medium (DMEM); these pigs were housed in a separate room from the MERS-CoV-inoculated pigs to prevent cross-contamination. Animal experiments were approved by the Institutional Animal Care and Use Committee of the Rocky Mountain Laboratories and conducted by certified staff in an Association for Assessment and Accreditation of Laboratory Animal Care International-accredited facility according to the institution's guidelines for animal use; staff followed the guidelines and basic principles in the US Public Health Service Policy on Humane Care and Use of Laboratory Animals and the Guide for the Care and Use of Laboratory Animals.

After inoculation with MERS-CoV, none of the pigs showed clinical signs of disease, such as increased body temperature or increased respiration, and bodyweight gain was similar between MERS-CoV-inoculated and mockinoculated pigs (Figure 2, panel A). We collected nose and throat swabs during clinical exams and analyzed them for the presence of viral RNA by quantitative reverse transcription PCR (qRT-PCR) as described (10). Shedding of viral RNA from the nose and the throat increased from 1 day postinoculation (dpi) to $3 \mathrm{dpi}$ in all MERS-CoV-inoculated animals, a sign that active replication occurred; 
Table. Comparison of the amino acid residues shown to be essential in binding of Middle East respiratory syndrome coronavirus spike protein to DPP4 of human, dromedary camel, and domestic pig*

\begin{tabular}{|c|c|c|c|c|c|c|c|c|c|c|c|c|c|c|}
\hline \multirow[b]{2}{*}{ Species } & \multicolumn{14}{|c|}{ DPP4, aa position } \\
\hline & 229 & 267 & 286 & 288 & 291 & 294 & 295 & 298 & 317 & 322 & 336 & 341 & 344 & 346 \\
\hline Human† & $\mathrm{N}$ & $\mathrm{K}$ & $\mathrm{Q}$ & $\mathrm{T}$ & $\mathrm{A}$ & $\mathrm{L}$ & $\mathrm{I}$ & $\mathrm{H}$ & $\mathrm{R}$ & $\mathrm{Y}$ & $\mathrm{R}$ & $\mathrm{V}$ & $Q$ & I \\
\hline Dromedary camelł & - & - & - & V & 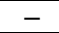 & - & - & - & - & - & - & - & - & 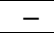 \\
\hline Domestic pig§ & - & - & - & $\mathrm{V}$ & - & - & - & - & - & - & - & - & - & - \\
\hline Mouseף & - & - & - & $\mathrm{P}$ & - & $\bar{A}$ & $\mathrm{R}$ & - & - & - & $T$ & $\mathrm{~S}$ & - & $\mathrm{V}$ \\
\hline $\begin{array}{l}\text { *DPP4, dipeptidyl pept } \\
\text { †GenBank accession } \\
\text { †GenBank accession } \\
\text { §GenBank accession } \\
\text { TTaken from previous }\end{array}$ & $\begin{array}{l}\text { ise 4; } \\
\text { AB45 } \\
\text { KF57 } \\
\text { NM21 } \\
\text { olicatic }\end{array}$ & $\begin{array}{l}10 \text { char } \\
39 . \\
3 . \\
57 . \\
7) .\end{array}$ & from & $\operatorname{nan} \mathrm{D}$ & & & & & & & & & & \\
\hline
\end{tabular}

shedding was higher in the nose than in the throat (Figure 2, panel B). After 3 dpi, shedding of viral RNA decreased; all nose swabs were negative by $11 \mathrm{dpi}$ and all throat swabs by 7 dpi.

We attempted virus propagation by inoculating VeroE6 cells with the media used to resuspend nasal and throat swab particulates and checking for the development of MERS$\mathrm{CoV}$ cytopathic effect. Infectious MERS-CoV was not recovered at any time postinoculation from any swab sample.
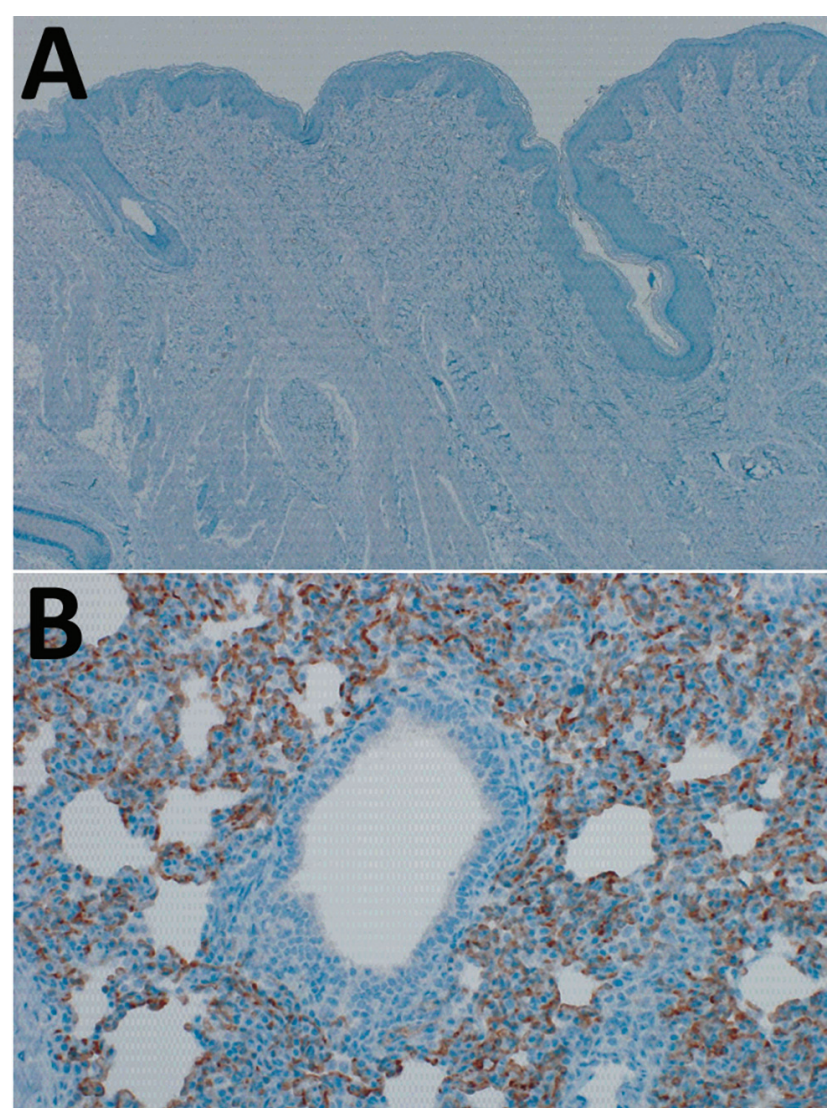

Figure 1. Dipeptidyl peptidase (DPP) 4 expression in the domestic pig respiratory tract. Tissues were stained by using a crossreactive mouse monoclonal antibody against DPP4 (CD26, clone OTI11D7, 1:2,500; Origene Technologies, Inc., Rockville, MD, USA). DPP4 expression was absent in the nasal mucosa (A) but present in lung tissue $(B)$ of healthy domestic pigs. Original magnification: nasal mucosa $\times 40$; lung $\times 200$.
On 4 and 11 dpi, we euthanized 4 MERS-CoV-inoculated pigs and collected their tissues for virologic and histologic analysis. Viral RNA could be detected by qRT-PCR in $\geq 1$ respiratory tract tissue samples of all 4 MERS-CoV-inoculated pigs. However, viral loads were low; infectious MERS-CoV could not be isolated from any tissues positive by qRT-PCR, and the distribution of viral RNA among tissues was inconsistent from pig to pig (Figure 2 panel C). By $11 \mathrm{dpi}$, viral RNA could only be detected in the bronchial lymph node of 1 MERS-CoVinoculated pig (Figure 2 panel C); all the tissues examined from other MERS-CoV-inoculated pigs were negative by this time. Viral RNA could not be detected in any of the extrarespiratory tissues tested, such as heart, liver, spleen, kidney, adrenal gland, duodenum, ileum, transverse colon, or urinary bladder, on 4 dpi or 11 dpi (data not shown). Histologic analysis did not reveal any lesions consistent with MERS-CoV infection in any of the collected tissues, including those of the respiratory tract. We performed immunohistochemical staining with an antibody specific for MERS-CoV on tonsil, trachea, bronchial lymph node, and right and left lower lung lobe of all pigs, as well as other tissues that tested positive for viral RNA by qRT-PCR. MERS-CoV antigen could not be detected in any of these tissues.

Serum samples collected on the day of euthanasia were tested for the presence of antibodies against MERS-CoV spike protein 1 (S1) by ELISA. By 11 dpi, antibodies directed against MERS-CoV S1 could be detected in all 4 pigs (Figure 2, panel D).

\section{Conclusions}

Recently, Vergara-Alert et al. showed MERS-CoV shedding in pigs inoculated with $10^{7} \mathrm{TCID}_{50}$ of MERS-CoV and suggested that pigs could play a role as a reservoir for the circulation of MERS-CoV (12). In our hands, pigs inoculated with a 10 -fold lower infectious dose of MERS-CoV were also successfully infected, but the low amount of virus replication in and shedding from the respiratory tract implies that the pig is unlikely to play a profound role as an intermediate host for MERS-CoV in nature. 


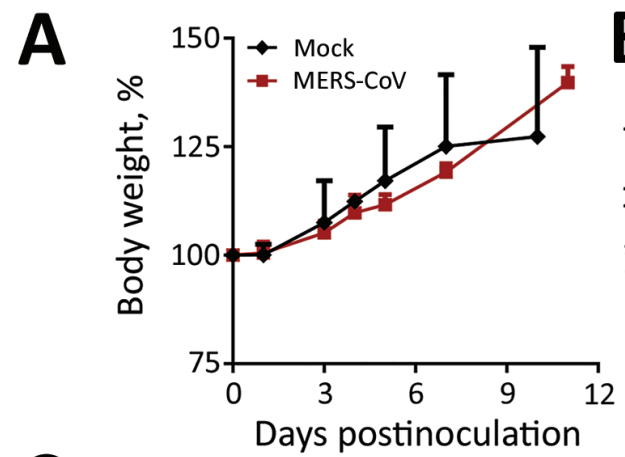

C

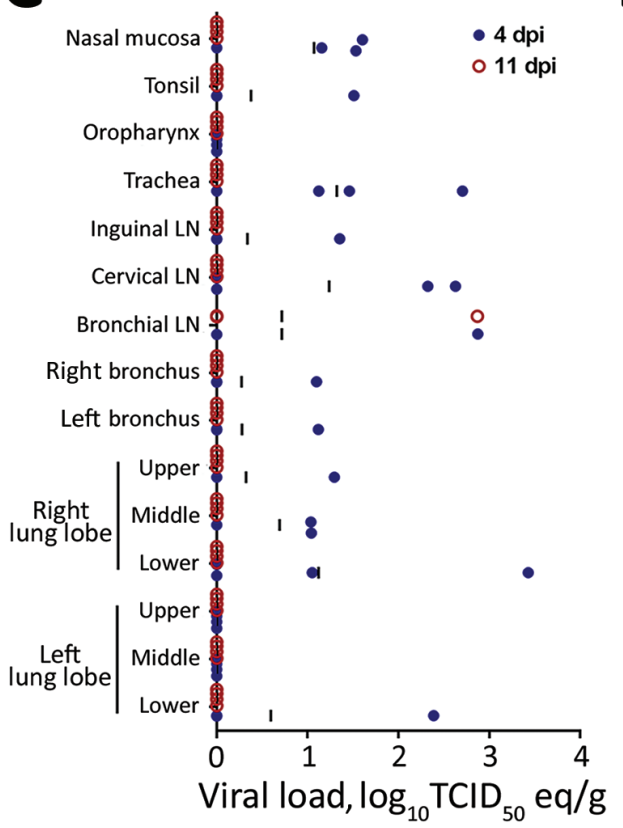

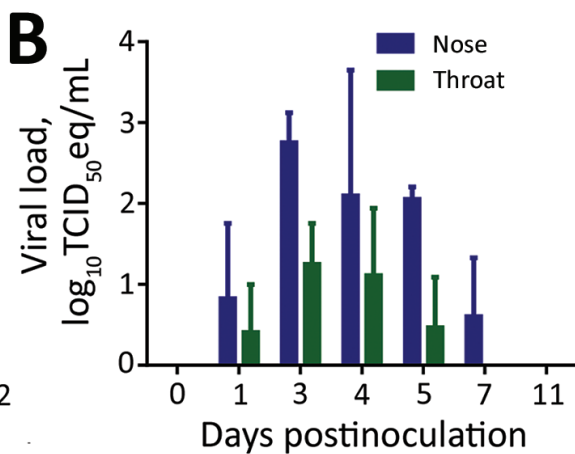

D

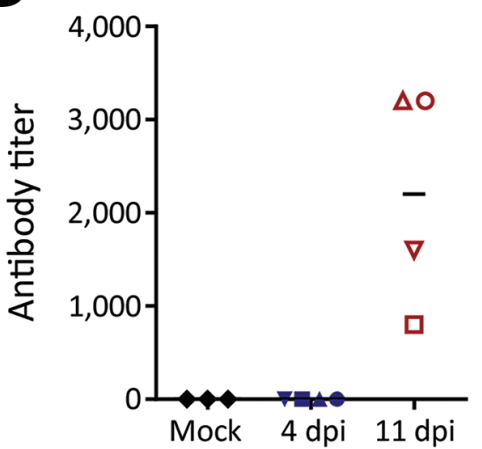

Figure 2. Propagation of Middle East respiratory syndrome coronavirus (MERS-CoV) in domestic pigs. We inoculated pigs intranasally and intratracheally with $10^{6}$ tissue culture infectious dose $50\left(\mathrm{TCID}_{50}\right)$ of MERS$\mathrm{CoV}$ isolate hCoV-EMC/2012 or for controls (mock-inoculated), with Dulbecco's modified Eagle medium. A) Mean bodyweight gain comparison between mock-inoculated and MERSCoV-inoculated animals over time. Error bars indicate SDs. B) Mean viral loads shed from the nose and throat determined at the time points indicated by quantifying virus on nasal and throat swabs collected from MERS-CoVinoculated animals using quantitative reverse transcription PCR (11); in each run, standard dilutions of a titered virus stock were run in parallel to calculate $\mathrm{TCID}_{50}$ equivalents. Error bars indicate SDs. C) Viral loads in tissues collected from MERS-CoV-inoculated animals on days 4 (closed blue circles) and 11 (open red circles) postinoculation. Viral loads were determined as in panel B. Vertical bars indicate means. D) Serum samples collected from pigs at the time of euthanasia (days 4 and 11 postinoculation) and tested for MERSCoV antibodies by using an ELISA for MERS-CoV spike 1 protein. Antibody titers are plotted as the reciprocal of the last serum dilution positive by ELISA. Horizontal bars indicate means. LN, lymph node.
Taken together, our data indicate that MERS-CoV can infect pigs, leading to a low level of replication in the pig respiratory tract, but does not cause clinical signs of disease. Furthermore, viral shedding from mucosal membranes of the upper respiratory tract was rather limited with no infectious virus measurable at any time postinoculation. Thus, the pig is not a suitable animal disease model for MERS-CoV infection.

\section{Acknowledgments}

The authors thank the Rocky Mountain Veterinary Branch staff for the care and handling of the animals, Anita Mora for help with figure preparation, and Juergen Richt for helpful advice.

This work was supported by the Intramural Research Program of the National Institute of Allergy and Infectious Diseases of the National Institutes of Health.

Dr. de Wit is a staff scientist in the Disease Modeling and Transmission section of the Laboratory of Virology. Her research interests are in the pathogenesis and transmission of emerging viruses.

\section{References}

1. World Health Organization. Middle East respiratory syndrome coronavirus (MERS-CoV)-Saudi Arabia. Disease outbreak news. 2017 Mar 10 [cited 2017 Mar 15]. http://www.who.int/csr/don/ 10-march-2017-mers-saudi-arabia/en/

2. Ng DL, Al Hosani F, Keating MK, Gerber SI, Jones TL, Metcalfe MG, et al. Clinicopathologic, immunohistochemical, and ultrastructural findings of a fatal case of Middle East respiratory syndrome coronavirus infection in the United Arab Emirates, April 2014. Am J Pathol. 2016;186:652-8. http://dx.doi.org/ 10.1016/j.ajpath.2015.10.024

3. Munster VJ, de Wit E, Feldmann H. Pneumonia from human coronavirus in a macaque model. N Engl J Med. 2013;368:1560-2. http://dx.doi.org/10.1056/NEJMc1215691

4. Baseler L, de Wit E, Feldmann H. A comparative review of animal models of Middle East respiratory syndrome coronavirus infection. Vet Pathol. 2016;53:521-31. http://dx.doi.org/ 10.1177/0300985815620845

5. Meurens F, Summerfield A, Nauwynck H, Saif L, Gerdts V. The pig: a model for human infectious diseases. Trends Microbiol. 2012;20:50-7. http://dx.doi.org/10.1016/j.tim.2011.11.002

6. Müller MA, Raj VS, Muth D, Meyer B, Kallies S, Smits SL, et al. Human coronavirus EMC does not require the SARS-coronavirus receptor and maintains broad replicative capability in mammalian cell lines. MBio. 2012;3:e0515-12. http://dx.doi.org/10.1128/ mBio.00515-12 
7. van Doremalen N, Munster VJ. Animal models of Middle East respiratory syndrome coronavirus infection. Antiviral Res. 2015;122:28-38. http://dx.doi.org/10.1016/j.antiviral.2015.07.005

8. Lu G, Hu Y, Wang Q, Qi J, Gao F, Li Y, et al. Molecular basis of binding between novel human coronavirus MERS-CoV and its receptor CD26. Nature. 2013;500:227-31. http://dx.doi.org/ 10.1038 /nature 12328

9. Wang N, Shi X, Jiang L, Zhang S, Wang D, Tong P, et al. Structure of MERS-CoV spike receptor-binding domain complexed with human receptor DPP4. Cell Res. 2013;23:986-93. http://dx.doi.org/10.1038/cr.2013.92

10. Corman VM, Eckerle I, Bleicker T, Zaki A, Landt O, Eschbach-Bludau M, et al. Detection of a novel human coronavirus by real-time reverse-transcription polymerase chain reaction. Euro Surveill. 2012;17:20285.
11. de Wit E, Rasmussen AL, Falzarano D, Bushmaker T, Feldmann F, Brining DL, et al. Middle East respiratory syndrome coronavirus (MERS-CoV) causes transient lower respiratory tract infection in rhesus macaques. Proc Natl Acad Sci U S A. 2013;110:16598-603. http://dx.doi.org/10.1073/pnas.1310744110

12. Vergara-Alert J, van den Brand JM, Widagdo W, Muñoz M, Raj S, Schipper D, et al. Livestock susceptibility to infection with Middle East respiratory syndrome coronavirus. Emerg Infect Dis. 2017;23:232-40. http://dx.doi.org/10.3201/eid2302.161239

Address for correspondence: Heinz Feldmann, Laboratory of Virology,

Division of Intramural Research, National Institute of Allergy and Infectious Diseases, National Institutes of Health, Rocky Mountain Laboratories, $903 \mathrm{~S}$ 4th St, Hamilton, MT 59840, USA; email: feldmannh@niaid.nih.gov

\section{December 2011 : Zoonotic Infections}

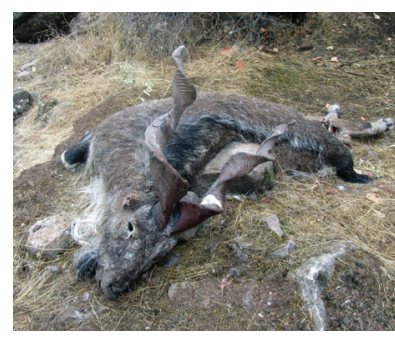

- Risk for Rabies Importation from North Africa

- Continuing Threat of Influenza (H5N1) Virus Circulation in Egypt

- Worldwide Occurrence and Impact of Human Trichinellosis, 1986-2009

- Sealpox Virus in Marine Mammal Rehabilitation Facilities, North America, 2007-2009

- Transmission of Guanarito and Pirital Viruses among Wild Rodents, Venezuela

- Hepatitis E Virus in Rats, Los Angeles, California

- Enterovirus Co-infections and Onychomadesis after Hand, Foot, and Mouth Disease, Spain

- Experimental Infection of Horses with Hendra Virus/ Australia/Horse/2008/ Redlands

- West Nile Virus Infection of Birds, Mexico
- Lineage and Virulence of Streptococcus suis Serotype 2 Isolates from North America

- Isolation of Prion with BSE Properties from Farmed Goat

- Candidate Cell Substrates, Vaccine Production, and Transmissible Spongiform Encephalopathies

- Molecular Epidemiology of Rift Valley Fever Virus

- Novel Multiplexed HIV/ Simian Immunodeficiency Virus Antibody Detection Assay

- Astroviruses in Rabbits

- Host Genetic Variants and Influenza-associated Mortality among Children and Young Adults

- Severe Human Bocavirus Infection, Germany

- Hepatitis E Virus Antibodies in Blood Donors, France

- Human Cardioviruses, Meningitis, and Sudden Infant Death Syndrome in Children

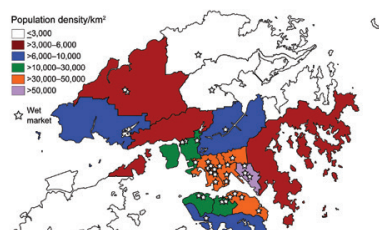

- Seroprevalence of Alkhurma and Other Hemorrhagic Fever Viruses, Saudi Arabia

- Knowledge of Avian Influenza (H5N1) among Poultry Workers, Hong Kong, China

- Risk for Human African Trypanosomiasis, Central Africa, 2000-2009

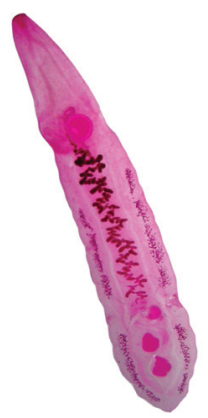

- Animal Diseases Caused by Orbiviruses, Algeria

- Genogroup I and II Picobirnaviruses in Respiratory Tracts of Pigs

- Human Liver Infection by Amphimerus spp. Flukes, Ecuador

- Q Fever in Woolsorters, Belgium

- Aedes aegypti Mosquitoes Imported into the Netherlands, 2010

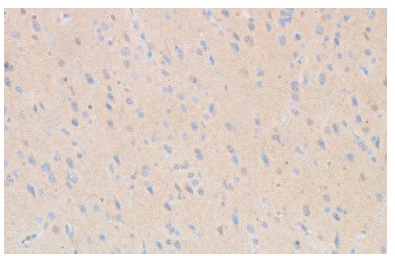

- Fatal Outbreak of Mycoplasma capricolum Pneumonia in Endangered Markhors

- African Swine Fever Virus Caucasus Isolate in European Wild Boars

- Novel Sylvatic Rabies Virus Variant in Endangered Golden Palm Civet, Sri Lanka

- Rickettsia parkeri in Amblyomma maculatum Ticks, North Carolina, 2009-2010

- Japanese Encephalitis Virus Genotype Replacement, Taiwan, 2009-2010

- Altitude-dependent Bartonella quintana Genotype C in Head Lice, Ethiopia

- Proximity to Goat Farms and Coxiella burnetii Seroprevalence among Pregnant Women

- Hemoptysis Associated with Leptospirosis Acquired in Hawaii 